\title{
Control Synthesis versus Saturation Compensation for Systems with Rate and Amplitude Constraints
}

\author{
K. Hui and C.W. Chan \\ Department of Mechanical Engineering, University of Hong Kong, Pokfulam Road, Hong Kong \\ Email: khui@hkumea.hku.hk \\ URL: http://hkumea.hku.hk/khui
}

\begin{abstract}
A control synthesis theory was proposed by Horowitz [2] to design a 'three degrees of freedom' controller for rate and amplitude constrained systems. Following anti-reset windup techniques, a saturation compensation structure was proposed to design compensators for given linear controllers [3]. It is shown here that the compensator can be reformulated in terms of the control synthesis theory. Conversely, the 'three degrees of freedom' controller is a special case of the compensator construction. From this analysis, shortcomings of the control synthesis theory are exposed and improvements using the compensator structure are discussed and illustrated by an example.
\end{abstract}

\section{Introduction}

Practical control systerns often encounter both rate and amplitude constraints from the actuators, reflecting physical bounds on finite power and energy transfers. In the literature, there are a felw discussions on systems subject to both rate and amplitude constraints $[2,5,7]$, with rate and amplitude constraints taken into account at the beginning of the controller designs. Alternative approach is to extend general actuator saturation compensators [4] for single constraints to systems subject to both rate and amplitude constraints [3]. Some recent theories of general anti-reset windup (ARW) [6,8] considered multiple saturation nonlinearities in parallel only, instead of in series as in rate and amplitude constrained systems.

A 'three degrees of freedom' controller structure was proposed by Horowitz to handle both rate and amplitude constraints [2]. Quantitative feedback theory (QFT) was then used to design the controller to cater for the constraints and plant uncertainties. The synthesis theory was later extended to unstable plants [7] by restricting the controller outputs to within bounds. To this purpose a supervisory loop was introduced to adjust a nonlinear gain, inserted between the nominal controller and the actuator. Frequency domain techniques were used to design the nominal controller.

The work in [5] considered the structure of amplitude nonlinearity before the rate nonlinearity. It also introduced a supervisory loop to adjust a nonlinear gain, called the error governor, in the closed-loop system. The error governor was inserted before the controller and adjusts the system error according to predetermined bounds.

These works are viewed as control synthesis techniques as they are for controller design or to predetermine the control bounds. A compensator for both rate and amplitude constraints was proposed in [3]. Conditions for stability of the compensated system were derived and some guidelines for designing the compensators discussed. ARW methods are a posteriori techniques in that the compensators are fabricated for given controllers, which were designed assuming absence of saturation. The relationship between these two approaches is not yet available.

In this work, the compensator structure [3] is reviewed in $\S 2$. The 'three degrees of freedom' control synthesis [2] is presented and reformulated as compensator structure in $\S 3$. It turns out to be a special case of the compensator discussed in $\S 2$. Compensator design methods for these two approaches are discussed next in $\S 4$, revealing some shortcomings of the synthesis theory. An illustrative example is presented in $\$ 5$.

\section{A Compensator Structure}

Let $G$ be the transfer function of a plant, and the linear controller be described by

$$
v=(T / R) w-(S / R) y
$$

where $y$ is the system output, $w$ is the reference input and $v$ is the controller output. $R, S, T$ are polynomials in Laplace transform variable $s$ or backward shift operator $z^{-1} . R$ is monic. The arguments are omitted for convenience. The amplitude and rate constraints for the actuator are

$$
\begin{gathered}
u(t)=\left\{\begin{array}{lr}
u_{\max }, & v \geq u_{\max } \\
v, & u_{\min } \leq v \leq u_{\max } \\
u_{\min }, & v \leq u_{\min }
\end{array}\right. \\
\dot{u}(t)=\left\{\begin{array}{lr}
\dot{u}_{\max }, & \dot{v} \geq \dot{u}_{\max } \\
\dot{v}, & \dot{u}_{\min } \leq \dot{v} \leq \dot{u}_{\max } \\
\dot{u}_{\min }, & \dot{v} \leq \dot{u}_{\min }
\end{array}\right.
\end{gathered}
$$

where $\left\{u_{\max }, u_{\sin }\right\}$ are the amplitude limits, and $\left\{\dot{u}_{\max }\right.$, $\left.\dot{u}_{\text {min }}\right\}$ the rate limits, respectively.

Due to difficulties in measuring the actuator velocity, an actuator model is inserted after the controller and before the actuator (c.f. [5,7]) with known bounds of $\left\{u_{\max }, u_{\min }\right\}$ and $\left\{\dot{u}_{\max }, \dot{u}_{\min }\right\}$. In this manner, both the velocity and the amplitude outputs of the actuator model, $\{u, \dot{u}\}$, are within the rate and amplitude bounds of the actuator. Saturation compensation is carried out within the actuator model.

Extending an ARW compensation framework for single-nonlinearity systems [4], rate and amplitude constrained system is compensated by two linear blocks: rate saturation by $P_{r}$, and amplitude saturation by $P_{a}$ [Fig.1]. $R_{2}$ models a differentiator $\left[R_{2}(s)=s\right]$. The separation of rate and amplitude constraints allows independent 
consideration and compensation of saturation effects.

From Fig. 1, the output of the actuator model is [3]

$v=(T / R) w-(S / R) y+\left[\left(1+P_{r}\right) / R_{2}\right] \delta_{r}+P_{a} \delta_{a}$

$u=(T / R) w-(S / R) y+\left[\left(1+P_{r}\right) / R_{2}\right] \delta_{r}+\left[1+P_{a}\right] \delta_{a}$

and $\quad \delta_{a}(t) \Delta u(t)-v(t) ; \delta_{r}(t) \Delta \dot{u}(t)-\dot{v}(t)$

For convenience, let $G_{C}=G S / R$ and

$$
\begin{aligned}
u_{0} & =\left[(T / R) /\left(1+G_{C}\right)\right] w \\
\Delta u_{r} & =\left[\left(1+P_{r}\right) / R_{2} /\left(1+G_{C}\right)\right] \delta_{r} \\
\Delta u_{a} & =\left[\left(1+P_{a}\right) /\left(1+G_{C}\right)\right] \delta_{a}
\end{aligned}
$$

then the closed-loop actuator output is

$$
u=u_{0}+\Delta u_{r}+\Delta u_{a}
$$

and the system output is

$$
\left.\begin{array}{l}
y=G u \underline{\Delta} y_{0}+\Delta y_{r}+\Delta y_{a} \\
y_{0}=G u_{0}, \Delta y_{r}=G \Delta u_{r}, \Delta y_{a}=G \Delta u_{a}
\end{array}\right\}
$$

$\left\{y_{0}, u_{0}\right\}$ are the unconstrained linear system responses. $\Delta y_{a}$ and $\Delta u_{a}$ arise from amplitude saturation, $\Delta y_{r}$ and $\Delta u_{r}$ from rate saturation. When there is no compensation, i.e., $P_{a}=P_{r}=0, y$ in (2.8) is still affected by $\Delta y_{a}$ and $\Delta y_{r}$, as $\delta_{a}$ and $\delta_{r}$ are nonzero. The fact that output $y$ is a linear function of these two saturations justifies separate compensation treatments for the rate and amplitude constraints.

Similar to single nonlinearity systems [4], the compensators need to satisfy the following conditions:

(P1) $P_{a}\left(z^{-1}\right)$ and $P_{r}\left(z^{-1}\right)$ each has at least one unit delay; or $P_{a}(s)$ and $P_{r}(s)$ are proper.

(P2) poles of $R P_{a}$ and $R\left(1+P_{r}\right) / R_{2}$ are asymptotically stable.

(P1) is the realizability condition, ensuring that the compensators are physically implementable. (P2) is necessary for $\Delta y_{r}$ and $\Delta y_{a}$ in (2.8) to be asymptotically stable and without steady state offsets. $G$ is assumed stable, as unstable $G$ cannot be globally stabilized [1].

To study the stability of rate and amplitude saturation compensated systems, equivalent systems representing Fig.1 are derived first. From (2.4)-(2.6), the following closed-loop expressions are obtained:

where

$$
\left.\begin{array}{l}
v=u_{0}+\Delta u_{r}+\Delta v_{a} \\
u_{1}=u_{10}+\Delta u_{1 r}+\Delta u_{1 a} \\
v_{1}=u_{10}+\Delta v_{1 r}+\Delta u_{1 a}
\end{array}\right\}
$$

$$
\left.\begin{array}{l}
\Delta v_{a}=\left[\left(P_{a}-G_{C}\right) /\left(1+G_{C}\right)\right] \delta_{a}, u_{10}=\left[T / R_{1} /\left(1+G_{C}\right)\right] w \\
\Delta u_{1 r}=\left[\left(1+P_{r}\right) /\left(1+G_{C}\right)\right] \delta_{r}, \Delta v_{1 r}=\left[\left(P_{r}-G_{C}\right) /\left(1+G_{C}\right)\right] \delta_{r} \\
\Delta u_{1 a}=-\left[G_{C} R_{2}\left(1+P_{a}\right) /\left(1+G_{C}\right)\right] \delta_{a}, R=R_{1} R_{2}
\end{array}\right\}
$$

From (2.7a) and (2.9), $\delta_{r}$ and $\delta_{a}$ are reconstructed from observations of $u$ and $u_{1}$ as

$$
\left.\begin{array}{c}
\delta_{a}=\left[1 /\left(1+P_{a}\right)\right] u-\left[\left(1 / R_{2}\right) /\left(1+P_{a}\right)\right] u_{\mathrm{I}} \\
\delta_{r}=\left[G_{C} R_{2} /\left(1+P_{r}\right)\right] u+\left[1 /\left(1+P_{r}\right)\right] u_{1} \\
-\left[\left(T / R_{1}\right) /\left(1+P_{r}\right)\right] w
\end{array}\right\}
$$

Using (2.10), (2.9) simplifies to

$$
\left.\begin{array}{lr}
v_{1}= & F_{w} w+G_{4} u_{1}-G_{2} u \\
v= & G_{1} u_{1}+G_{3} u
\end{array}\right\}
$$

where the equivalent blocks are

$$
\begin{aligned}
& G_{1}=\left(1 / R_{2}\right) /\left(1+P_{a}\right), G_{2}=\left(G S / R_{1}\right) /\left(1+P_{r}\right) \\
& G_{3}=P_{a} /\left(1+P_{a}\right) \quad, G_{4}=P_{r} /\left(1+P_{r}\right) \\
& F_{w}=\left(T / R_{1}\right) /\left(1+P_{r}\right), R=R_{1} R_{2}
\end{aligned}
$$

(2.11) describes an equivalent configuration of Fig.1, shown in Fig.2, where $\mathrm{N}_{\mathrm{r}}$ represents the rate constraint nonlinearity and $\mathrm{N}_{\mathrm{a}}$ the amplitude constraint nonlinearity. As $\left\{G_{1}, G_{2}, G_{3}, G_{4}\right\}$ in (2.12) have to be open-loop stable for the compensated system to be globally stabilizable [1], $\left\{P_{a}, P_{r}\right\}$ must satisfy the additional condition:

(P3) zeros of $\left[1+P_{a}\right]$ and $\left[1+P_{r}\right]$ are asymptotically stable. For nonlinear stability analysis [3], let $\mathrm{N}_{\mathrm{r}}$ be replaced by its dynamic gain $k_{r}$ and $\mathrm{N}_{\mathrm{a}}$ by $k_{a}, 0<k_{a}, k_{r} \leq \mathrm{l}$. Then the characteristic equation of the saturation compensated system shown in Fig.2 is [3]

$$
\left[1+\left(1-k_{a}\right) P_{a}\right]\left[1+\left(1-k_{r}\right) P_{r}\right]+k_{a} k_{r} G_{C}=0
$$

If $k_{a}=k_{r} \equiv 1$, then (2.13) degenerates to the unconstrained linear system: $1+G_{C}=0$. Furthermore, the equivalent system $G_{k a}$ as seen by the amplitude constraint nonlinearity, is obtained by writing (2.13) in the form of

$$
\left.\begin{array}{l}
1+k_{a} G_{E a}=0 \\
G_{E a}=\left\{\left(k_{r} G_{C}\right) /\left[1+\left(1-k_{r}\right) P_{r}\right]-P_{a}\right\} /\left(1+P_{a}\right)
\end{array}\right\}
$$

and that seen by the rate constraint nonlinearity is

$$
\left.\begin{array}{l}
1+k_{r} G_{E r}=0 \\
G_{E r}=\left\{\left(k_{a} G_{C}\right) /\left[1+\left(1-k_{a}\right) P_{a}\right]-P_{r}\right\} /\left(1+P_{r}\right)
\end{array}\right\}
$$

Assessments of nonlinear stability of the rate and amplitude constrained systems using (2.13)-(2.14) can be found in [3].

\section{A Control Synthesis Theory}

The control synthesis theory proposed by Horowitz [2] is now reviewed. To compare the two approaches, the synthesis theory is reformulated into the structure of $\$ 2$.

The system configuration used by Horowitz [2] is reproduced in Fig.3. Without loss of generality, noise disturbances are omitted here and the same notations as in Fig. 1 are used. Plant constituents $\left\{P_{1}, P_{2}, P_{3}, P_{4}\right\}$ are assumed known prior to the control synthesis process. $\left\{F_{n}\right.$, $\left.G_{n}, H_{n}\right\}$ are the 'three degrees of freedom' of the controller and are to be synthesized. From Fig.3,

$$
v_{1}=P_{3}\left[P_{4} G_{n}\left(F_{n} w-y\right)-H_{n} P_{2} u\right]
$$

and from (2.6)

$$
u=v+\delta_{a}, v=\left(1 / R_{2}\right) u_{1}, u_{1}=v_{1}+\delta_{r}
$$

(3.1) can be written as

$$
\left.\begin{array}{c}
v_{1}=\frac{P_{3} P_{4} G_{n}}{1+H_{n} P_{2} P_{3} / R_{2}}\left(F_{n} w-y\right) \\
-\frac{H_{n} P_{2} P_{3} / R_{2}}{1+H_{n} P_{2} P_{3} / R_{2}} \delta_{r}-\frac{H_{n} P_{2} P_{3}}{1+H_{n} P_{2} P_{3} / R_{2}} \delta_{a}
\end{array}\right\}
$$




$$
\left.\begin{array}{l}
v=\frac{P_{3} P_{4} G_{n} / R_{2}}{1+H_{n} P_{2} P_{3} / R_{2}}\left(F_{n} w-y\right) \\
+\frac{1 / R_{2}}{1+H_{n} P_{2} P_{3} / R_{2}} \delta_{r}-\frac{H_{n} P_{2} P_{3} / R_{2}}{1+H_{n} P_{2} P_{3} / R_{2}} \delta_{a}
\end{array}\right\}
$$

Comparing (3.4) with the compensated controller in (2.4), the following relationships are obtained:

$$
\begin{aligned}
& \qquad \frac{S}{R} \triangleq \frac{P_{3} P_{4} G_{n} / R_{2}}{1+H_{n} P_{2} P_{3} / R_{2}} ; \frac{T}{R} \triangleq \frac{S F_{n}}{R} \\
& \frac{1+P_{r}}{R_{2}} \triangleq \frac{1 / R_{2}}{1+H_{n} P_{2} P_{3} / R_{2}} ; P_{a} \Delta \frac{-H_{n} P_{2} P_{3} / R_{2}}{1+H_{n} P_{2} P_{3} / R_{2}} \\
& \text { From (3.6): } \quad P_{a} \equiv P_{r} \\
& \text { Plant } G \text { is given by } \quad G \quad \triangleq P_{1} P_{2}
\end{aligned}
$$

That is, the direct control synthesis by Horowitz [2] is to determine the controller transfer functions $S / R, T / R$ and simultaneously define a common compensator $P_{a}$ or $P_{r}$ for both rate and amplitude saturations. Any modifications to $\left\{F_{m}, G_{n}, H_{n}\right\}$ change $\{R, S, T\}$ and $\left\{P_{a}, P_{r}\right\}$ simultaneously. The loop transmission $G_{C}$ and the closed-loop transfer function $H$ are respectively [2]:

$$
P_{n} \Delta \frac{P_{1} P_{2} P_{3} P_{4}}{R_{2}}, G_{C}=\frac{P_{n} G_{n}}{1+H_{n} P_{2} P_{3} / R_{2}}, H=\frac{F_{n} G_{C}}{1+G_{C}}
$$

From (3.5)-(3.7), the direct control synthesis [2] is clearly a special case of the compensator discussed in $\$ 2$. In other words, a posteriori compensator designs may be treated as an a priori synthesis method. As $P_{a}=P_{r}$, then

$$
L_{n} \triangleq G_{E r}\left(k_{a}=1\right)=G_{E a}\left(k_{r}=1\right)
$$

$L_{n}$ plays an important role in the control synthesis, where the design consideration was developed from the governing equation (5) in [2]:

$\Delta v_{1} \Delta v_{1}-v_{10}, \Delta u \Delta u-u_{0}: \Delta v_{1}=-R_{2} L_{n} \Delta u$

Based on (3.11), it was suggested to adopt [2]

$$
L_{n}(s)=\left(2 \xi \omega_{n} s+\omega_{n}^{2}\right) / s^{2}
$$

Given $\left\{P_{1}, P_{2}, P_{3}, P_{4}\right\}$, the design methodology was to:

(i) choose $\left\{G_{C}, H\right\}$ according to QFT and $L_{n}$ as (3.12);

(ii) calculate controller $\left\{F_{n}, G_{n}, H_{n}\right\}$ from

$$
F_{n}=\frac{H\left(1+G_{C}\right)}{G_{C}}, G_{n}=\frac{G_{C}\left(1+L_{n}\right)}{P_{n}\left(1+G_{C}\right)}, H_{n}=\frac{R_{2}\left(L_{n}-G_{C}\right)}{P_{2} P_{3}\left(1+G_{C}\right)}
$$

The bulk of the design work was in the specifications of $\left\{G_{C}, H, L_{n}\right\}$ using QFT, instead of on $\left\{F_{n}, G_{n}, H_{n}\right\}$. The equivalence for controller in (3.5)-(3.6) are

$$
\begin{aligned}
& S / R=G_{C} / P_{1} P_{2}, T / R=H\left(1+G_{C}\right) / P_{1} P_{2} \\
& P_{a}=P_{r}=\left(G_{C}-L_{n}\right) /\left(1+L_{n}\right)
\end{aligned}
$$

An insight can be obtained comparing (3.11), and the corresponding relationship derived from the closed-loop quantities in $\S 2$. From (2.7a),

$$
\begin{gathered}
\Delta u=\Delta u_{r}+\Delta u_{a}=\left[1 /\left(1+G_{E}\right)\right]\left(\delta_{r} / R_{2}+\delta_{a}\right) \\
\text { giving } \quad \delta_{r} / R_{2}+\delta_{a}=\left(1+G_{E}\right) \Delta u
\end{gathered}
$$

since $P_{a}=P_{r}$ and denote $G_{l}=G_{l: r}\left(k_{a}=1\right)=G_{l: a}\left(k_{r}=1\right)$. From (2.7b) and using (3.16)

$$
\Delta v_{1}=R_{2}\left[\frac{P_{r}-G_{C}}{1+G_{C}}\right]\left(1+G_{E}\right) \Delta u-R_{2} P_{a} \delta_{a}
$$

$$
\text { giving } \quad \Delta v_{1}=-R_{2} G_{E} \Delta u-R_{2} P_{a} \delta_{a}
$$

Comparing (3.18) with (3.11), the design basis in [2] was for the special condition: $\quad R_{2} P_{a} \delta_{a}=0$ Therefore, the design proposed by Horowitz is mainly for systems dominated by velocity constraints, i.e., $\left|\delta_{r}\right|>>\left|\delta_{a}\right|$. The success of Horowitz's design method is due to the fact that normally the rate constraint, being ahead of the amplitude constraint, comes with more pronounced effects. Subsequently the use of (3.11), instead of (3.18), approximates the system behaviours reasonably well.

One drawback of (3.12) is that the system must be of sufficiently low order; otherwise the implementation of controller (3.13) becomes a practical problem.

\section{Compensator Design}

The effects of rate and amplitude constraints on a control system are fundamentally different, e.g., amplitude constrained PID controllers give rise to overshoots and long settling times, while rate constrained PID controllers yield sluggish and nonminimum phase responses [4]. Therefore, the same compensator cannot generally be used for both rate and amplitude constraints, as the compensators can perform well under either rate or amplitude constraints only. Since the system under consideration is rate constrained first, it is logical to expect that performance of the rate compensator $P_{r}$ would have a more pronounced effect on the system performance, than that by the amplitude compensator $P_{a}$. This was implicitly utilized by Horowitz's synthesis. A design procedure to select $\left\{P_{a}, P_{r}\right\}$ is discussed below.

From (2.14), the equivalent systems may be written as

$$
\begin{aligned}
1+G_{E i a}=F_{a}\left[1+G_{a}\right] ; F_{a}=\frac{1}{1+P_{a}}, G_{a}=\frac{k_{r} G_{C}}{1+\left(1-k_{r}\right) P_{r}} \\
1+G_{E r}=F_{r}\left[1+G_{r}\right] ; F_{r}=\frac{1}{1+P_{r}}, G_{r}=\frac{k_{a} G_{C}}{1+\left(1-k_{a}\right) P_{a}}
\end{aligned}
$$

An interpretation of (4.1) is that the Nyquist curve of $1+G_{E a}(j \omega)$ is the same as that of $\left[1+G_{a}(j \omega)\right]$ modified by $F_{a}(j \omega)$, i.e., $P_{a}$ modifies behaviours of the Nyquist curves of $\left[1+G_{E a}(j \omega)\right]$ via $F_{a}(j \omega)$. Consequently, compensator design can be performed by proper selection of $F_{a}$. The same interpretation holds for $F_{r}$ in (4.2) with respect to the rate saturation compensation. Due to the symmetry, the following discussions only consider the design of $F_{a}$.

Assume that for a chosen $\left\{P_{a 0}, P_{r}\right\}$-pair, the stability behaviour of the compensated system $G_{E a}$ is not satisfactory and an improved compensator $P_{a 1}$ is needed. Let $G_{E a 1}$ be the desired compensated system. Then the $\left[1+G_{E a 1}(j \omega)\right]-$ plot is given by reshaping the $\left[1+G_{E a}(j \omega)\right]$-plot with a filter $F(j \omega)$ : 
$1+G_{E a 1}=F\left[1+G_{E a}\right]=\frac{F\left[1+G_{a}\right]}{1+P_{a 0}}=\frac{1+G_{a}}{1+P_{a 1}}$

in which the later equalities are from (4.1). The modified compensator is thus given by

$$
P_{a 1}=\left[1+P_{a 0}\right] / F-1
$$

If $P_{a 0}=0$, then (4.4) amounts to the direct design of $P_{a}$. A feasible lead filter design for $F$ is

$$
F(s)=\frac{s+1 / \beta}{s+1 / \alpha \beta} \quad ; \alpha=\frac{1+\sin \phi}{1+\sin \phi}, \beta=\frac{1}{\omega_{m} \sqrt{\alpha}}
$$

so that $F(s)$ introduces a phase shift $\phi$ at frequency $\omega_{m}$. The digital equivalent of (4.5) was shown in [4]. $P_{a 1}$ is checked to satisfy (P1)-(P3). $\left\{\phi, \omega_{m}\right\}$ are determined as follows.

Assuming the $G_{E a}(j \omega)$-plot as in Fig.4, interception of $G_{E a}(j \omega)$-plot with the -ve real-axis implies local stability only [1]. To improve the stability behaviours, point $\mathrm{A}$ as shown in Fig. 4 may be identified. Let its frequency be $\omega_{0}$ and $\angle \mathrm{ACB}=\varphi_{0}$, choose

$$
\omega_{m}=\omega_{0} \text { and } \phi=\varphi_{0}+\Delta \phi
$$

with $\Delta \phi \approx 0.5^{\circ} \sim 10^{\circ}$, filter $F$ is thus completely defined and so is the modified compensator (4.4). Exact amount of $\Delta \phi$ may be fined tuned when necessary.

\section{Design Procedure}

(1) Choose $P_{a}=0$ or $P_{r}=0$.

(2) Plot $G_{E a}\left(j \omega ; k_{r}\right)$ of (2.14a) for $0 \leq k_{r} \leq 1$, or $G_{E r}\left(j \omega ; k_{a}\right)$ of (2.14b) for $0 \leq k_{a} \leq 1$.

(3) If none of Nyquist curves intercept to the left of $-1+j 0$ on the real axis, and the compensated system response is satisfactory, exit design loop.

(4) If interception exists, identify the appropriate phase angle and frequency such as Fig. 4.

(5) Calculate filter (4.5) and compensator (4.4).

(6) If the compensated system response is satisfactory, exit design loop. Else return to Step (2).

The above procedure is first used to design the rate compensator $P_{r}$ and then the amplitude compensator $P_{a}$. Finally the above procedure is used, if necessary, to select $P_{a}$ and $P_{r}$ simultaneously for modifications of the preliminary compensators, in order to ensure that necessary conditions for global stability are satisfied [1].

Apart from choosing $P_{a}=0$ and $P_{r}=0$ in Step (1), they can be initialized using existing compensation schemes $[4,6,8]$. In such case the above Design Procedure can be used to ensure fulfillment of necessary and/or sufficient conditions for global asymptotic stability.

\section{Example}

The following example was studied by Horowitz [2] and will be compared here between the control synthesis and the compensator design technique. The improved performance of the compensated system with simpler designs supports the compensator approach.

From [2], plant $G(s)=1 / s$, and the controller is

$$
\frac{S(s)}{R(s)}=\frac{T(s)}{R(s)}=\frac{13(s+5)^{3}}{(s+0.7)^{3}(s / 250+1)}
$$

The uncompensated system limit-cycles when subject to constraints $\left\{u_{\text {limit }}= \pm 1 ; \dot{u}_{\text {limit }}= \pm 0.5\right\}$, as shown in phase diagrams $[e=y-w ; \dot{e}=d e / d t]$ of Fig.5 for unit step inputs.

Using (3.12) with $\xi=1$ and $\omega_{n}=16$, the control synthesis gives the following high order controllers of $G_{n}$ and $H_{n}$ from (3.13) $\left[F_{n}=1\right]$ as

$$
\left.\begin{array}{c}
G_{n}=3250(s+5)^{3}(s+16)^{2} / D_{n} \\
H_{n}=32\left[s^{5}+158.5 s^{4}+1019.8 s^{3}+3037.6 s^{2}\right. \\
-9666.8 s+686] / D_{n}
\end{array}\right\}
$$

Clearly such complex transfer functions are highly unsatisfactory from implementation considerations. The equivalent lead filter $F_{r}(s)$ [from (3.14) and (4.2)] is

$$
F_{r}=(s+0.7)^{3}(s+16)^{2}(s+250) / s D_{n}
$$

To design the rate and amplitude compensators, a $G_{C}(j \omega)$ plot reveals that $\left\{\varphi_{0}=57.7^{\circ} ; \omega_{0}=1.9\right\}$ [Fig.4]. Thus $\phi$ may take $60^{\circ}$ and both $G_{E \alpha}(j \omega)$ - and $G_{E r}(j \omega)$-plots are to advance this amount by the compensators. From (4.4)-(4.5), the amplitude saturation compensator $P_{a}$ is designed as

$$
P_{a}(s)=6.6122 /(s+0.5115)
$$

To design the rate compensator $P_{r}(s)$, notice that (5.1) does not have an integrator and so, in order to satisfy condition $(\mathbf{P 2}), F_{r}(s)$ must include one which however introduces a $90^{\circ}$ phase lag into $\angle F_{r}(j \omega)$. Consequently a cascade of $(4.5)$ is required to shift $150^{\circ}$ in $\angle F_{r}(j \omega)\left[90^{\circ}+60^{\circ}=150^{\circ}=2 \times 75^{\circ}\right]$. Choose two lead filters of $75^{\circ}$ each, and then replace one pole by an integrator to satisfy (P1-P2), giving

$$
\begin{aligned}
& F_{r}(s)=(s+0.25)^{2} / s(s+14.93) \\
& P_{r}(s)=(13.93 s-0.0625) /(s+0.25)^{2}
\end{aligned}
$$

The compensators $\{(5.4),(5.6)\}$ are applied and the results shown in Figs.6-7. Improvements in the system responses are clearly observed in comparison with those obtained by the control synthesis using (5.2). This system is dominated by velocity constraints as $\left|\delta_{r}\right|>>\left|\delta_{a}\right|$ [Fig.5]. For more severe saturation levels, it will be necessary to further advance $F_{r}(s)$ and then higher order compensators cannot be avoided. Incidentally, the phase shift of (5.3) is $71.3^{\circ}$ at $\omega_{\mathrm{m}}=1.9$, and its maximum is $72.7^{\circ}$ at $\omega_{\mathrm{m}}=2.36$.

\section{Conclusion}

This paper compares a control synthesis theory proposed by Horowitz for designing controllers for systems subject to rate and amplitude constraints, and a compensator structure previously proposed for given linear controllers. It was shown that the control synthesis theory is a special case of the compensator construction. In a limited scope, it shows the equivalence between a priori control synthesis and $a$ 
posteriori compensator designs. It also shows that the design selection proposed by Horowitz was only appropriate for low-order saturation systems dominated by velocity constraints, whereas the compensator structure applies to any combination of the severity of rate and amplitude constraints. Results were demonstrated with an illustrative example.

\section{References}

[1] Atherton, D.P. Nonlinear Control Engineering, 1982 Van Nostrand Reinhold

[2] Horowitz, I., Feedback systems with rate and amplitude limiting, Int. J. Control vol.40 no.6 pp.1215-1229 1984

[3] Hui, K. and C.W. Chan, A Stability Analysis of Controllers subject to Amplitude and Rate Constraints, Proc. IEEE Int. Conf. Cont. App. pp.259-264 1997

[4] Hui, K. and C.W. Chan, New Design Methods of Actuator Saturation Compensators for PID Controllers, J. Syst. Cont. Engg. vol.211 n.I4 pp.269-280 1997

[5] Kapasouris, P. and M. Athans, Control Systems with Rate and Magnitude Saturation for Neutrally Stable Open Loop Systems, Proc. 29th IEEE CDC pp.3404-3409 1990

[6] Kothare, M.V., P.J. Campo, M. Morari and C.N. Nett, Unified framework for the study of anti-windup designs, Automatica vol.30 n.12 pp.1869-1883 1994

[7] Liao, Y.K. and I.M. Horowitz, Unstable uncertain plants with rate and amplitude saturations, Int. J. Control vol.44 no.4 pp.1147-1159 1986

[8] Walgama, K.S. and J.Sternby, Conditioing technique for multiinput multioutput processes with input saturation, IEE Proc.-D vol.140 no. 4 pp.231-241 1993

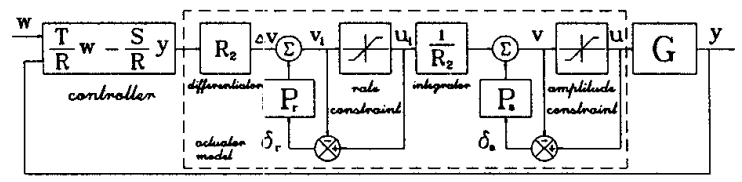

Fig.1 Rate and amplitude saturation compensated system

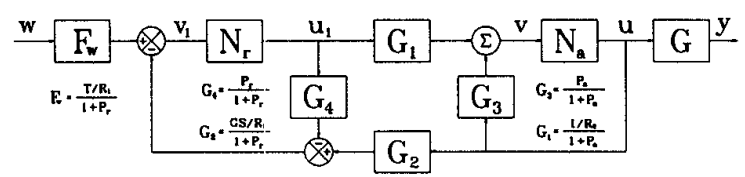

Fig.2 Equivalent system of Fig. 1 for stability analysis

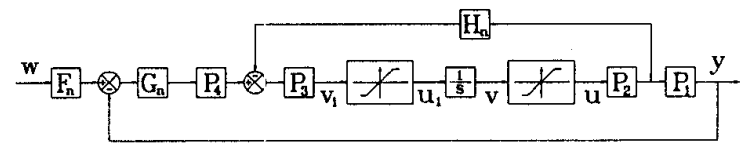

Fig.3 Controller structure from Horowitz [2]

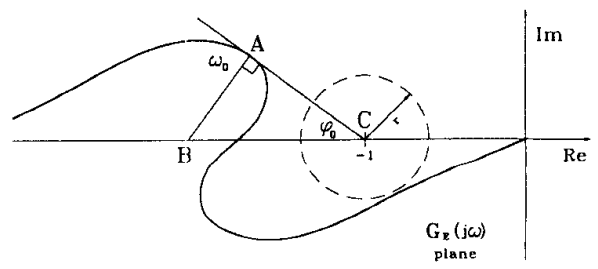

Fig.4 Determination of filter parameters

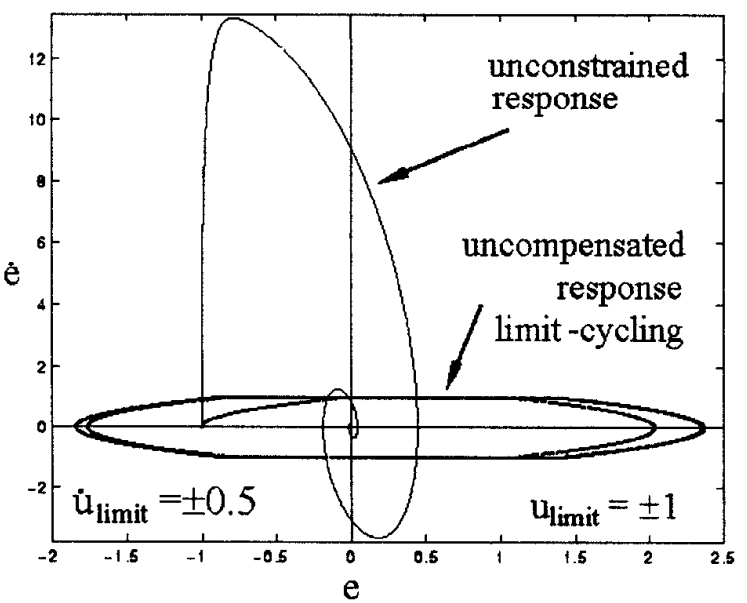

Fig.5 Phase diagram for unit step inputs. Velocity saturation dominates: $\left|\delta_{r}\right|_{\max }=3249.5$ versus $\mid \delta_{a \mid \max }=0.56$.

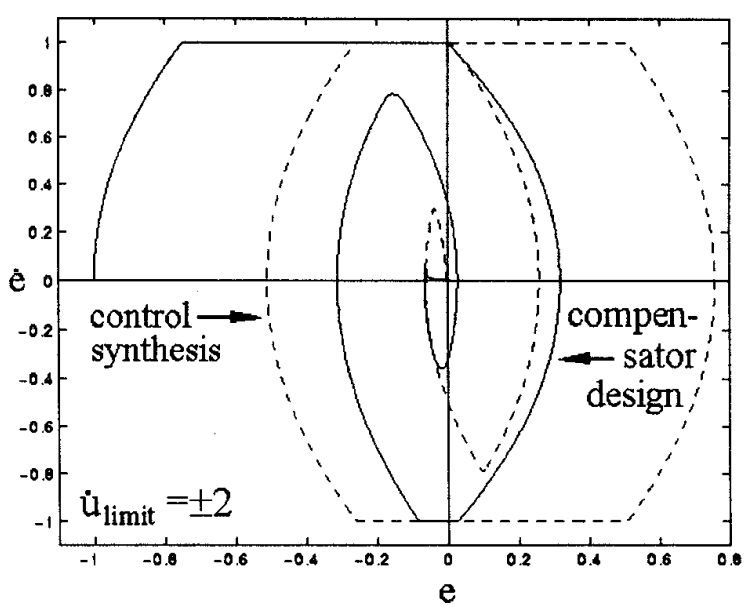

Fig.6 Stable compensated responses @ $\dot{u}_{\text {limit }}= \pm 2$

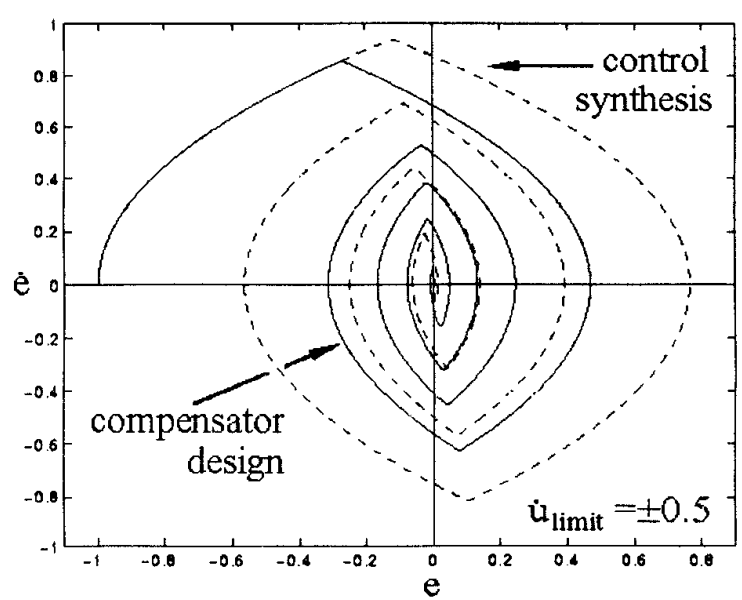

Fig.7 Stable compensated responses@ $\dot{u}_{\text {limit }}= \pm 0.5$ 